\title{
Detection of signal recognition particle (SRP) RNAs in the nuclear ribosomal internal transcribed spacer I (ITSI) of three lineages of ectomycorrhizal fungi (Agaricomycetes, Basidiomycota)
}

\author{
Magnus Alm Rosenblad', María P. Martín², Leho Tedersoo ${ }^{3}$, Martin Ryberg$^{4}$,
} Ellen Larsson ${ }^{5}$, Christian Wurzbacher ${ }^{5}$, Kessy Abarenkov ${ }^{6}$, R. Henrik Nilsson ${ }^{5}$

I Department of Marine Sciences, University of Gothenburg, Bioinformatics Infrastructure for Life Sciences, Box 460, SE-405 30 Göteborg 2 Departmento de Micología, Real Jardin Botánico, RJB-CSIC, Plaza de Murillo 1, 28014 Madrid, Spain 3 Institute of Ecology and Earth Sciences, University of Tartu, Tartu, Estonia 4 Department of Organismal Biology, Evolutionary Biology Centre, Uppsala University, Sweden 5 Department of Biological and Environmental Sciences, University of Gothenburg, Box 463, 40530 Göteborg, Sweden 6 Natural History Museum, University of Tartu, Vanemuise 46, Tartu 510 14, Estonia

Corresponding author: R. Henrik Nilsson (henrik.nilsson@bioenv.gu.se)

Academic editor: T. Lumbsch | Received 22 March 2016 | Accepted 21 April 2016 | Published 13 May 2016

Citation: Rosenblad MA, Martín MP, Tedersoo L, Ryberg M, Larsson E, Wurzbacher C, Abarenkov K, Nilsson RH (2016) Detection of signal recognition particle (SRP) RNAs in the nuclear ribosomal internal transcribed spacer 1 (ITS1) of three lineages of ectomycorrhizal fungi (Agaricomycetes, Basidiomycota). MycoKeys 13: 21-33. doi: 10.3897/ mycokeys.13.8579

\begin{abstract}
During a routine scan for Signal Recognition Particle (SRP) RNAs in eukaryotic sequences, we surprisingly found in silico evidence in GenBank for a 265-base long SRP RNA sequence in the ITS1 region of a total of 11 fully identified species in three ectomycorrhizal genera of the Basidiomycota (Fungi): Astraeus, Russula, and Lactarius. To rule out sequence artifacts, one specimen from a species indicated to have the SRP RNA-containing ITS region in each of these genera was ordered and re-sequenced. Sequences identical to the corresponding GenBank entries were recovered, or in the case of a non-original but conspecific specimen differed by three bases, showing that these species indeed have an SRP RNA sequence incorporated into their ITS1 region. Other than the ribosomal genes, this is the first known case of non-coding RNAs in the eukaryotic ITS region, and it may assist in the examination of other types of insertions in fungal genomes.
\end{abstract}

\section{Key words}

SRP RNA, non-coding RNA, ribosomal RNA, internal transcribed spacer 1 (ITS1), ectomycorrhizal fungi

Copyright Magnus Alm Rosenblad et al. This is an open access article distributed under the terms of the Creative Commons Attribution License (CC BY 4.0), which permits unrestricted use, distribution, and reproduction in any medium, provided the original author and source are credited. 


\section{Introduction}

The nuclear ribosomal internal transcribed spacer (ITS) region is part of the ribosomal DNA cistron. The ITS region is transcribed together with the $18 \mathrm{~S}, 5.8 \mathrm{~S}$, and $28 \mathrm{~S}$ genes but removed in the post-transcriptional processing of the rRNA. The ITS region has three separate subregions: the ITS1, the $5.8 \mathrm{~S}$ gene, and the ITS2. The ITS1 is situated between the $18 \mathrm{~S}$ and the $5.8 \mathrm{~S}$ genes, and the ITS2 is situated between the $5.8 \mathrm{~S}$ and the $28 \mathrm{~S}$ genes. The ITS region varies significantly in length among fungal species (Taylor and McCormick 2008, Tedersoo et al. 2015), and both of ITS1 and ITS2 form secondary structures with stems, bulges, and loops (Freire et al. 2012, Rampersad 2014). The secondary structure is important for correct processing of the rRNA; although the ITS1 and ITS2 regions are not expressed in the ribosome, there are constraints on the evolution of the ITS region, and it has both fast evolving and more conserved regions (Nazar 2004, Mullineaux and Hausner 2009). However, there is still much to learn about the function of the ITS region, especially for ITS1 (Rampersad 2014, Coleman 2015). The fast evolving regions of ITS has made it a cornerstone in species/genuslevel phylogenetic inference in fungi and other organisms for more than 20 years, and it is the formal fungal barcode used for molecular species identification (Álvarez and Wendel 2003, Schoch et al. 2012).

One element that has never been implicated in the context of the eukaryotic rDNA cluster and ITS evolution is the existence of non-coding RNAs (ncRNA) other than the $18 \mathrm{~S}, 5.8 \mathrm{~S}$, and $28 \mathrm{~S}$ rRNAs. Based on the recent identification of a ubiquitous eukaryotic ncRNA in the fungal phylum Basidiomycota, viz. the Signal Recognition Particle RNA (SRP RNA; Dumesic et al. 2015), we discovered a -265 bases long homologue of this gene in a set of fungal ITS1 sequences (Fig. 1). The SRP RNA is an essential component of the SRP, a ribonucleoprotein particle that co-translationally directs proteins to the endoplasmic reticulum (ER) membrane. The SRP RNA acts both as a scaffold for the SRP proteins and as a regulator of the SRP by mediating a global reorganization of the SRP in response to cargo binding (Rosenblad et al. 2009, Akopian et al. 2013).

A more thorough in silico analysis verified the presence of SRP RNAs in the ITS1 region of a total of 11 fully identified fungal species (separate Latin binomials) distributed over three lineages of ectomycorrhizal basidiomycetes (Boletales: Astraeus (1 species: A. sirindhorniae), Russulales: Russula (1 species: R. olivacea), and Russulales: Lactarius (9 species: L. argillaceifolius, L. aspideus, L. brunneoviolaceus, L. luridus, L. nanus, L. pallescens, L. pseudouvidus, L. uvidus, and L. violascens). The notion of an additional ncRNA element in the ITS1 region is novel and would seem - at least at a first glance - to compromise the function of the ITS1. Hypothetically, any of contamination, chimeric unions, or other laboratory or data analysis artifacts could explain this finding. In this study we apply DNA sequencing and bioinformatics to verify the presence of SRP RNA sequences in the ITS1 region of representatives of these fungi. 


\section{Materials and methods}

\section{SRP RNA bioinformatics}

The bioinformatic analysis of non-coding RNAs such as the SRP RNA is not trivial, as the primary sequence may vary substantially as long as the secondary structure is preserved. To enable searches for SRP RNAs without requiring exact sequence matches across the full length of the SRP RNA, we used a secondary structure covariance model constructed from the full set of available ascomycete SRP RNAs with the basidiomycete SRP RNAs from Dumesic et al. (2015) added, as well as a second dataset containing all covariance models from Rfam (Nawrocki et al. 2014). These models were used in an INFERNAL v1.1 cmsearch (Nawrocki and Eddy 2013) run against flatfiles of the International Nucleotide Sequence Database Collaboration (INSDC; Nakamura et al. 2013; February 2015 release). After observing several highly significant matches to what seemed to be the fungal ITS1 region, we re-ran the search on the flatfiles from the manually curated fungal ITS database UNITE (Abarenkov et al. 2010; release 2015-08-01) using ITSx 1.0.11 (Bengtsson-Palme et al. 2013) to identify the ITS1 region. A total of 63 matches and 11 fully identified species from three ectomycorrhizal basidiomycete lineages were recovered (Suppl. material 1). All matches were examined manually to verify that they displayed all the universally conserved motifs and nucleotides. The sequences were found to stem from more than 20 different published and unpublished studies. The fact that these sequences had been found multiple times independently is highly suggestive of technically sound, authentic sequence data (Nilsson et al. 2012), but to further confirm the authenticity of the sequences we re-sequenced one herbarium specimen from each of these lineages, either from the original material or from other conspecific specimens.

\section{PCR and sequencing}

To rule out systematic PCR artifacts as sources of false positives in the bioinformatics analyses, we retrieved the original, or conspecific, specimens underlying one representative from each of the genera (Table 1): the conspecific collection Astraeus sirindhorniae MA-Fungi 47735 (collected in the Philippines; herbarium MA), the authentic collection Lactarius luridus TU118993 (collected in Estonia; herbarium TU), and the authentic collection Russula olivacea TU101845 (collected in Estonia; herbarium TU). We specifically sought to use a different primer pair combination and PCR conditions than did the original sequence authors in a further attempt at generalizing our findings. For $A$. sirindhorniae, DNA extractions, PCR reactions, and sequencing were performed as described in Martín and Winka (2000), however using DNeasy Plant Mini Kit (Qiagen) with overnight incubation for DNA extraction. Primers used for amplification were ITS5/ITS4 (White et al. 1990; Suppl. material 1). For the specimens of 
Lactarius and Russula, the DNA was extracted and amplified using the primers ITS1f and ITS4b following Anslan and Tedersoo (2015). Sequences were edited and assembled using Sequencher 4.2 (Gene Codes, Ann Arbor). All sequences were examined for sequence quality following Nilsson et al. (2012). Chimera detection was undertaken using UCHIME (Edgar et al. 2011) and the UNITE chimera reference dataset (Nilsson et al. 2015; release 2015-03-11).

\section{Results}

The ITS sequences recovered from the sequencing round passed all quality control measures we exercised. In addition, no sequence was found to have the multiple DNA ambiguity symbols suggestive of the presence of several information-wise distinct ITS copies in the individuals at hand (Hyde et al. 2013). The resulting sequences manifested the SRP RNA sequence in the ITS1 region of all three re-sequenced lineages. The two authentic specimens of Russula and Lactarius produced identical ITS sequences to those already extant. The sequence from the conspecific Astraeus sirindhorniae specimen differed by three bases from the extant sequence, which is well within the expected intraspecific variation when conspecific isolates are compared across geographical distances (Thailand and the Philippines in this case). The sequences were deposited in the INSDC as accessions KU356730-KU356732.

SRP RNA-containing sequences of Russula and Lactarius were found to have an average length of some 890 bases; the corresponding average length for the SRP RNAcontaining Astraeus sequences was 840 bases. When using BLAST to find the most similar sequences of Russula, Lactarius, and Astraeus that did not contain the SRP RNA, we found that their ITS region was on average 616 bases (Russula), 644 bases (Lactarius), and 620 bases (Astraeus). This corresponds well to the length of the SRP RNA ( -265 bases) for all of Astraeus, Russula, and Lactarius, allowing for some few bases of divergence considering the cross-species comparison. The distances between the SRP RNA and the surrounding genes $18 \mathrm{~S}$ and $5.8 \mathrm{~S}$ were almost the same within each lineage, but differed somewhat among the three lineages: 80 and 174 bases ( Lactarius), 150 and 80 bases (Russula), and 55 and 132 bases (Astraeus).

\section{Discussion}

The finding that ncRNAs are located in tandem is not novel. Apart from the highly conserved nuclear rDNA cluster, some ncRNAs have been found to cluster in several protist species, e.g., SRP RNA together with U6 snRNA, 5S rRNA, SL RNA, and tRNAs in dinoflagellates (Zhang et al. 2013). Regarding the transcription of the SRP RNA in fungi, the transcriptional promoters of the SRP RNA in Saccharomyces cerevisiae (the TFIIIC-binding A- and B-box) are internal, and the SRP RNAs have a polypyrimidine termination sequence similar to other RNA polymerase III genes. Although 


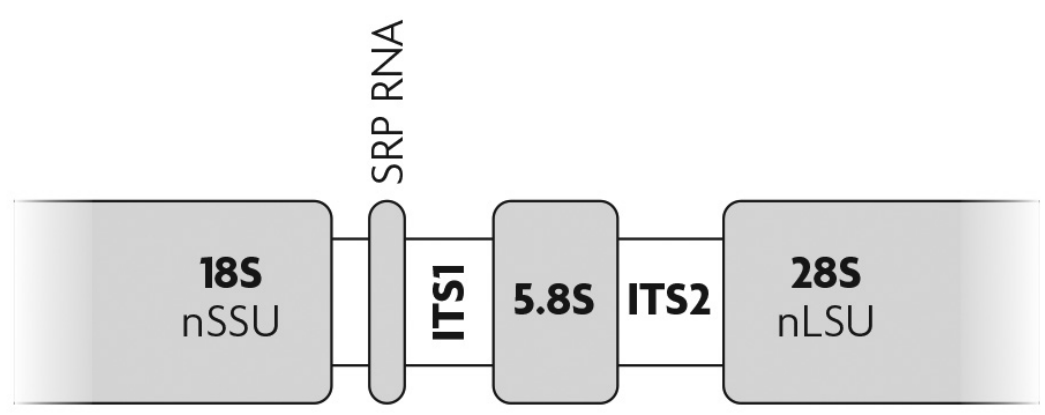

Figure I. Schematic illustration of the fungal ITS region and neighboring rDNA genes. The subregions ITS1, 5.8S, and ITS2 of the ITS region are indicated along with the SRP RNA in the first part of the ITS1. The absolute positions of the subregions and the SRP RNA are provided in Suppl. material 2.

yeast SRP RNA also has an upstream TATA box - a motif we could not clearly identify in our alignments - inactivation of this region does not result in a significant effect on transcription (Dieci et al. 2002). Therefore it is possible that our identified SRP RNAs could be transcribed independently of the transcription of the rDNA cluster. If so, the SRP RNAs found in the ITS1 region most probably are fully functional, but there may be another copy in the genome that constitutes the major transcript. Whether the SRP RNA could be a product of the rDNA processing seems less likely. Although the $\mathrm{A}_{3}$ cleavage site that is the closest to the $5.8 \mathrm{~S}$ should be downstream of the SRP RNA 3' end since the distance between the SRP RNA and the 5.8S is at least 80 nucleotides in the 63 sequences, the SRP RNA needs correct 5' and 3' ends to fold into the proper secondary structure. Therefore the processing of the tricistronic ribosomal transcript most probably leads to non-functional SRP RNA. The surprising lack of mutations, as compared to other identified basidiomycete SRP RNAs (Suppl. material 4), could be explained not only by the insertion being a recent event, but also by the need to preserve the secondary structure of the SRP RNA region and thus the remaining parts of ITS1.

The three species from which the SRP RNA was recovered are all ectomycorrhizal basidiomycetes and come from two different orders and two different families. Two of these lineages are closely related (Russula and Lactarius, both in Russulaceae (Russulales)); the third one - Astraeus sirindhorniae (Boletales) - comes from the same subphylum (Agaricomycotina) as the former two. Even so, the Russulales and the Boletales are separate orders, such that the presence of SRP RNAs in these fungi must be considered independent gains. In the case of Russula and Lactarius - two very speciose genera - the vast majority of the known species do not have the SRP RNAs in their ITS1. Similarly, none of the other species in Astraeus treated by Phosri et al. (2014) were found to have the SRP RNA. It would appear far more realistic to view these SRP RNAs as independent insertion events than as a plesiomorphic ITS state where the ancestor contained the SRP RNA, but where all species except the few considered here lost it (Miller et al. 2006; Suppl. materials 1-4). Indeed, we found no evidence for SRP RNA in the ITS region of any other fungus. 


\begin{tabular}{|c|c|c|c|c|}
\hline 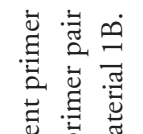 & ڤ્ & I & 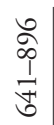 & $\begin{array}{l}\vec{a} \\
0 \\
b \\
0 \\
\sigma\end{array}$ \\
\hline 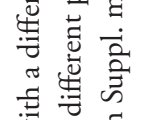 & $\begin{array}{l}\infty \\
\dot{n} \\
\dot{n}\end{array}$ & 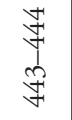 & 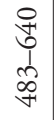 & 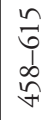 \\
\hline 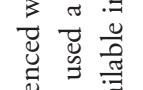 & 馬 & $\stackrel{\mathscr{F}}{\stackrel{4}{7}}$ & $\begin{array}{c}O S \\
\stackrel{+}{+} \\
-1\end{array}$ & $\hat{\tilde{f}}$ \\
\hline 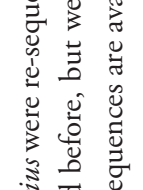 & 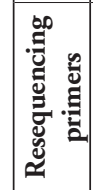 & 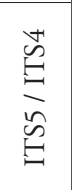 & 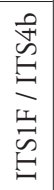 & 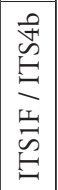 \\
\hline 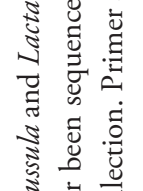 & 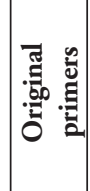 & 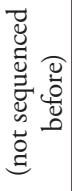 & 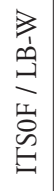 & 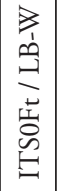 \\
\hline 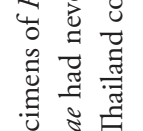 & 递 & 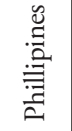 & 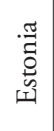 & 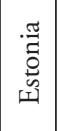 \\
\hline 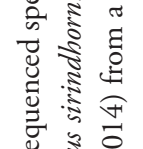 & 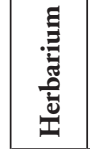 & $\frac{\bar{\pi}}{\sum^{\frac{\pi}{2}}}$ & $\underset{⿱ 乛 ⿻ コ 一 心 ~}{Z}$ & $\begin{array}{r}\vec{Z} \\
\text { 孛 }\end{array}$ \\
\hline 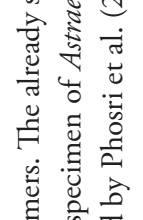 & $\begin{array}{l}\text { कू } \\
\text { के } \\
\text { के }\end{array}$ & 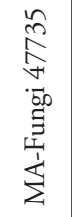 & 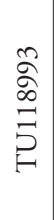 & $\begin{array}{l}n \\
+ \\
\infty \\
0 \\
0 \\
\vdots \\
1 \\
1\end{array} \mid$ \\
\hline 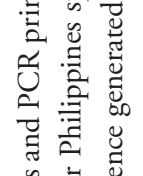 & 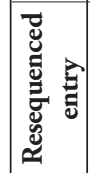 & 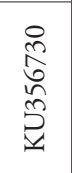 & $\begin{array}{l}\vec{n} \\
\hat{0} \\
\widehat{n} \\
\hat{v}\end{array}$ & $\begin{array}{l}\tilde{n} \\
\hat{\sigma} \\
n \\
\hat{n} \\
\vdots \\
\vdots\end{array}$ \\
\hline 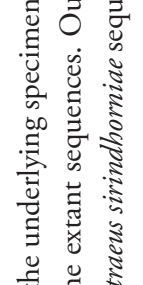 & 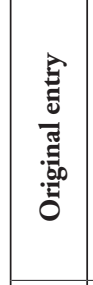 & 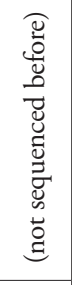 & 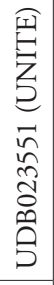 & 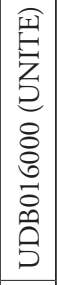 \\
\hline 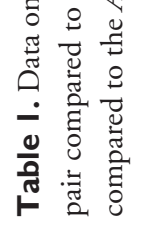 & के & 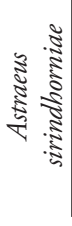 & 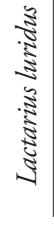 & 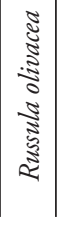 \\
\hline
\end{tabular}


The three previously identified SRP RNAs in the Russulales are not located in or close to the rDNA cluster (Dumesic et al. 2015), and we argue that the SRP RNA must be considered as an independently inserted element in these ITS1 sequences. Although this does not cause any problems in terms of molecular identification of these species, it does present a potential difficulty to the uncritical use of ITS sequences in phylogenetic inference in these fungal lineages. Under the assumption that the SRP RNA is found in the exact same position in the ITS1 region among species, the sequences could still be aligned jointly as long as the SRP RNA part is kept as a separate element in the multiple sequence alignment. Any failure to realize that the SRP RNA should be treated as a separate element to be scored as gaps in species that do not have the SRP RNA is certain to give rise to very noisy multiple sequence alignments and skewed inferences of phylogeny. In other words, there is a risk that alignment tools will try to align other parts of the ITS1 region onto the SRP RNA part in large alignments, which would violate homology assumptions. We briefly examined whether several of the most commonly used alignment programs were able to recognize the unique nature of the SRP RNA and not try to stack other parts of the ITS1 onto the SRP RNA. The results were generally encouraging as long as the number of non-SRP RNA containing species was kept reasonably low, with only minor manual adjustments needed a posteriori. In the worse situation where the SRP RNA insertion is not found in the exact same position across species, it will not be possible to maintain position homology in the multiple sequence alignment. In that scenario, the sequences containing the SRP RNA must be excluded from the alignment process, or the SRP RNA element must be removed. Interestingly, Eberhardt (2002) reported an unexpected 250-base insertion in the ITS1 of Russula olivacea - one of the species examined in the present study - and chose to exclude it from her multiple sequence alignment due to alignment difficulties. In hindsight it seems probable that this 250-base region indeed represents the SRP RNA. As demonstrated by the Eberhardt (2002) example, there is widespread (although not necessarily universal) awareness of the importance of examining multiple sequence alignments manually before they are put to scientific use. However, the increasing use of fully automated solutions to data harvesting and phylogenetic inference may present a concern here (cf. Antonelli et al. 2014).

Our findings are not without potential shortcomings though. The number of ITS copies per fungal cell can approach 200 or more (Bellemain et al. 2010; Black et al. 2013). Whereas the process of concerted evolution is thought to homogenize the array of ITS copies (Álvarez and Wendel 2003), it is not uncommon to find evidence of two or more distinct ITS copies during sequencing work (Hyde et al. 2013). A recent pyrosequencing-based study found evidence for multiple, information-wise distinct ITS copies in 3-5\% of the 99 examined species of Ascomycota and Basidiomycota (Lindner et al. 2013). The extent to which the three species examined here contain multiple distinct ITS copies is unknown but may well be low, given that we obtained single, clean PCR products and sequence chromatograms for all three species. Even so, it is conceivable that we - much like the original sequence authors - in fact amplified a rare and perhaps non-functional ITS copy. Although this would not disqualify our 
finding of an SRP RNA in the fungal ITS region, it would raise questions regarding whether the inclusion of the SRP RNA ruined the function of this particular ITS copy, essentially rendering the corresponding rRNA non-functional. In either case, we view this multiple-copy scenario as unlikely given our consistent obtainment, and the more than 20 independent recoveries, of the SRP RNA-containing ITS1 sequences.

Unfortunately, none of the species of the present study have a complete genome published, so a detailed analysis of the SRP RNA in the context of the genomes of these and closely related fungi will have to wait. That said, the trend that published fungal genomes tend to come without the ribosomal operon for reasons of convenience is most unfortunate (Schoch et al. 2014). We join the barcoding community in extending a plea that whenever a genome is sequenced and assembled, the ribosomal operon should be assembled into the genome as a part of that process. If this undertaking proves to be too complex, then at least the full ribosomal operon should be bundled with the genome, even if its assembly into the genome cannot be accomplished.

\section{Conclusions}

We found evidence of Signal Recognition Particle (SRP) RNAs in the ITS1 region of a total of 11 fully identified species in three ectomycorrhizal genera: Astraeus, Russula, and Lactarius. Other than the ribosomal genes, this is the first known case of noncoding RNAs in the fungal ITS region. Our finding is small step towards explaining the many insertions found throughout fungal genomes, and it adds a new element to the field of fungal ITS evolution.

\section{Acknowledgements}

Roy Watling is acknowledged for his Philippines collection of Astraeus sirindhorniae. Herbaria TU and MA are gratefully acknowledged for assistance with the specimens used in this study. RHN acknowledges financial support from FORMAS (215-2011498) and from Stiftelsen Olle Engkvist Byggmästare. MPM was partially supported by Plan Nacional I+D+i project CGL2012-35559. CW acknowledges a Marie Skłodowska-Curie post doc grant (660122, CRYPTRANS).

\section{References}

Abarenkov K, Nilsson RH, Larsson K-H, Alexander IJ, Eberhardt U, Erland S, Høiland K, Kjøller R, Larsson E, Pennanen T, et al. (2010) The UNITE database for molecular identification of fungi - recent updates and future perspectives. New Phytologist 186(2): 281-285. doi: $10.1111 /$ j.1469-8137.2009.03160.x 
Akopian D, Shen K, Zhang X, Shan SO (2013) Signal Recognition Particle: An essential protein targeting machine. Annual Review of Biochemistry 82: 693-721. doi: 10.1146/ annurev-biochem-072711-164732

Álvarez I, Wendel JF (2003) Ribosomal ITS sequences and plant phylogenetic inference. Molecular Phylogenetics and Evolution 29(3): 417-434. doi: 10.1016/S1055-7903(03)00208-2

Anslan S, Tedersoo L (2015) Performance of cytochrome c oxidase subunit I (COI), ribosomal DNA Large Subunit (LSU) and Internal Transcribed Spacer 2 (ITS2) in DNA barcoding of Collembola. European Journal of Soil Biology 69: 1-7. doi: 10.1016/j.ejsobi.2015.04.001 Antonelli A, Condamine FL, Hettling H, Nilsson K, Nilsson RH, Oxelman B, Sanderson MJ, Sauquet H, Scharn R, Silvestro D, et al. (2014) SUPERSMART: ecology and evolution in the era of big data. PeerJ PrePrints 2: e501v1. doi: 10.7287/peerj.preprints.501v1

Bellemain E, Carlsen T, Brochmann C, Coissac E, Taberlet P, Kauserud H (2010) ITS as an environmental DNA barcode for fungi: an in silico approach reveals potential PCR biases. BMC Microbiology 10: 189. doi: 10.1186/1471-2180-10-189

Bengtsson-Palme J, Ryberg M, Hartmann M, Branco S, Wang Z, Godhe A, De Wit P, Sánchez-García M, Ebersberger I, de Sousa F, et al. (2013) Improved software detection and extraction of ITS1 and ITS2 from ribosomal ITS sequences of fungi and other eukaryotes for analysis of environmental sequencing data. Methods in Ecology and Evolution 4810: 914-919. doi: 10.1111/2041-210X.12073

Black J, Dean T, Byfield G, Foarde K, Menetrez M (2013) Determining fungi rRNA copy number by PCR. Journal of Biomolecular Techniques 24(1): 32-38. doi: 10.7171/jbt.132401-004

Coleman AW (2015) Nuclear rRNA transcript processing versus internal transcribed spacer secondary structure. Trends in Genetics 31(3): 157-163. doi: 10.1016/j.tig.2015.01.002

Dieci G, Giuliodori S, Catellani M, Percudani R, Ottonello S (2002) Intragenic promoter adaptation and facilitated RNA polymerase III recycling in the transcription of SCR1, the 7SL RNA gene of Saccharomyces cerevisiae. Journal of Biological Chemistry 277(9): 6903-6914. doi: 10.1074/jbc.M105036200

Dumesic PA, Rosenblad MA, Samuelsson T, Nguyen T, Moresco JJ, Yates JR, Madhani HD (2015) Noncanoncial signal recognition particle RNAs in a major eukaryotic phylum revealed by purification of SRP from the human pathogen Cryptococcus neoformans. Nucleic Acids Research 43(18): 9017-9027. doi: 10.1093/nar/gkv819

Eberhardt U (2002) Molecular kinship analyses of the agaricoid Russulaceae: correspondence with mycorrhizal anatomy and sporocarp features in the genus Russula. Mycolological Progress 1(2): 201-223. doi: 10.1007/s11557-006-0019-6

Edgar RC, Haas BJ, Clemente JC, Quince C, Knight R (2011) UCHIME improves sensitivity and speed of chimera detection. Bioinformatics 27(16): 2194-2200. doi: 10.1093/bioinformatics/btr381

Freire MCM, da Silva MR, Zhang X, Almeida ÁMR, Stacey G, de Oliveira LO (2012) Nucleotide polymorphism in the $5.8 \mathrm{~S}$ nrDNA gene and internal transcribed spacers in Phakopsora pachyrhizi viewed from structural models. Fungal Genetics and Biology 49(2): 95-100. doi: 10.1016/j.fgb.2011.12.010 
Hyde KD, Udayanga D, Manamgoda DS, Tedersoo L, Larsson E, Abarenkov K, Bertrand YJK, Oxelman B, Hartmann M, Kauserud H, et al. (2013) Incorporating molecular data in fungal systematics: a guide for aspiring researchers. Current Research in Environmental and Applied Mycology 3(1): 1-32.

Katoh K, Standley DM (2013) MAFFT multiple sequence alignment software version 7: improvements in performance and usability. Molecular Biology and Evolution 30(4): 772-780. doi: $10.1093 / \mathrm{molbev} / \mathrm{mst} 010$

Lindner DL, Carlsen T, Nilsson RH, Davey M, Schumacher T, Kauserud H (2013) Employing 454 amplicon pyrosequencing to reveal intragenomic divergence in the internal transcribed spacer rDNA region in fungi. Ecology and Evolution 3(6): 1751-1764. doi: 10.1002/ece3.586

Maddison DR, Swofford DL, Maddison WP (1997) NEXUS: an extensible file format for systematic information. Systematic Biology 46(4): 590-621. doi: 10.1093/sysbio/46.4.590

Martín MP, Winka K (2000) Alternative methods of extracting and amplifying DNA from lichens. Lichenologist 32(2): 189-196. doi: 10.1006/lich.1999.0254

Miller SL, Larsson E, Larsson K-H, Verbeken A, Nuytinck J (2006) Perspectives in the new Russulales. Mycologia 98(6): 960-970. doi: 10.3852/mycologia.98.6.960

Mullineaux T, Hausner G (2009) Evolution of rDNA ITS1 and ITS2 sequences and RNA secondary structures within members of the fungal genera Grosmannia and Leptographium. Fungal Genetics and Biology 46(11): 855-867. doi: 10.1016/j.fgb.2009.08.001

Nakamura Y, Cochrane G, Karsch-Mizrachi I (2013) The international nucleotide sequence database collaboration. Nucleic Acids Research 41(D): D21-D24. doi: 10.1093/nar/gks1084

Nawrocki EP, Eddy SR (2013) Infernal 1.1: 100-fold faster RNA homology searches. Bioinformatics 29(22): 2933-2935. doi: 10.1093/bioinformatics/btt509

Nawrocki EP, Burge SW, Bateman A, Daub J, Eberhardt RY, Eddy SR, Floden EW, Gardner PP, Jones TA, Tate J, Finn RD (2014) Rfam 12.0: updates to the RNA families database. Nucleic Acids Research 43(D1): D130-D1373. doi: 10.1093/nar/gku1063

Nazar RN (2004) Ribosomal RNA processing and ribosome biogenesis in eukaryotes. IUBMB Life 56(8): 457-465. doi: 10.1080/15216540400010867

Nilsson RH, Tedersoo L, Abarenkov K, Ryberg M, Kristiansson E, Hartmann M, Schoch CL, Nylander JAA, Bergsten J, Porter TM, et al. (2012) Five simple guidelines for establishing basic authenticity and reliability of newly generated fungal ITS sequences. MycoKeys 4: 37-63. doi: 10.3897/mycokeys.4.3606

Nilsson RH, Tedersoo L, Ryberg M, Hartmann M, Unterseher M, Porter TM, BengtssonPalme J, Walker DM, de Sousa F, et al. (2015) A comprehensive, automatically updated fungal ITS sequence dataset for reference-based chimera control in environmental sequencing efforts. Microbes and Environments 30(2): 145-150. doi: 10.1264/jsme2.ME14121

Phosri C, Watling R, Suwannasai N, Wilson A, Martín MP (2014) A new representative of star-shaped fungi: Astraeus sirindhorniae sp. nov. from Thailand. PLoS ONE 9(5): e71160. doi: 10.1371/journal.pone.0071160

Rampersad SN (2014) ITS1, 5.8S and ITS2 secondary structure modelling for intra-specific differentiation among species of the Colletotrichum gloeosporioides sensu lato species complex. SpringerPlus 3: 684. doi: 10.1186/2193-1801-3-684 
Rosenblad MA, Larsen N, Samuelsson T, Zwieb C (2009) Kinship in the SRP RNA family. RNA Biology 6(5): 508-516. doi: 10.4161/rna.6.5.9753

Schoch CL, Seifert KA, Huhndorf S, Robert V, Spouge JL, Levesque CA, Chen W, et al. (2012) Nuclear ribosomal internal transcribed spacer (ITS) region as a universal DNA barcode marker for Fungi. Proceedings of the National Academy of Sciences USA 109(16): 6241-6246. doi: 10.1073/pnas.1117018109

Schoch CL, Robbertse B, Robert V, Vu D, Cardinali G, Irinyi L, Meyer W, Nilsson RH, Hughes K, Miller AN, et al. (2014) Finding needles in haystacks: linking scientific names, reference specimens and molecular data for Fungi. Database (Oxford) 2014: bau061. doi: 10.1093/database/bau061

Taylor DL, McCormick MK (2008) Internal transcribed spacer primers and sequences for improved characterization of basidiomycetous orchid mycorrhizas. New Phytologist 177(4): 1020-1033. doi: 10.1111/j.1469-8137.2007.02320.x

Tedersoo L, Anslan S, Bahram M, Pôlme S, Riit T, Liiv I, Kóljalg U, Kisand V, Nilsson H, Hildebrand F, Boork P, Abarenkov K (2015) Shotgun metagenomes and multiple primer pair-barcode combinations of amplicons reveal biases in metabarcoding analyses of fungi. MycoKeys 10: 1-43. doi: 10.3897/mycokeys.10.4852

White TJ, Bruns T, Lee S, Taylor JW (1990) Amplification and direct sequencing of fungal ribosomal RNA genes for phylogenetics. PCR protocols: a guide to methods and applications 18: 315-322.

Zhang H, Campbell DA, Sturm NR, Rosenblad MA, Dungan CF, Lin S (2013) Signal Recognition Particle RNA in dinoflagellates and the perkinsid Perkinsus marinus. Protist 164(5): 748-761. doi: 10.1016/j.protis.2013.07.004 


\section{Supplementary material I}

\section{Output from cmsearch and primers used}

Authors: Magnus Alm Rosenblad, María P. Martín, Leho Tedersoo, Martin Ryberg, Ellen Larsson, Christian Wurzbacher, Kessy Abarenkov, R. Henrik Nilsson

Data type: text/computer output

Explanation note: A) The output from cmsearch showing all 63 relevant matches to the three ectomycorrhizal lineages. B) Detail of the primers used to re-amplify the specimens.

Copyright notice: This dataset is made available under the Open Database License (http://opendatacommons.org/licenses/odbl/1.0/). The Open Database License $(\mathrm{ODbL})$ is a license agreement intended to allow users to freely share, modify, and use this Dataset while maintaining this same freedom for others, provided that the original source and author(s) are credited.

\section{Supplementary material 2}

\section{ITS multiple sequence alignment}

Authors: Magnus Alm Rosenblad, María P. Martín, Leho Tedersoo, Martin Ryberg, Ellen Larsson, Christian Wurzbacher, Kessy Abarenkov, R. Henrik Nilsson

Data type: text/DNA sequence data

Explanation note: A multiple sequence alignment in the NEXUS format (Maddison et al. 1997) comprising all 63 matching ITS sequences, plus the three newly generated ones (KU356730, KU356731, and KU356732). The alignment was produced in MAFFT without manual adjustment (Katoh and Standley 2013). The alignment is composed of partial nSSU (bases 1-34 in the alignment), the full ITS1 (bases 35-678), the full 5.8S (bases 679-838), the full ITS2 (bases 839-1395), and partial nLSU (bases 1396-end). The SRP RNA occupies position 203-474 in the alignment. The alignment is provided for overview purposes only; the two-order nature of the taxa (Boletales and Russulales) coupled with the high variability of the ITS region jointly mean that the alignment will not be suited for phylogenetic inference.

Copyright notice: This dataset is made available under the Open Database License (http://opendatacommons.org/licenses/odbl/1.0/). The Open Database License $(\mathrm{ODbL})$ is a license agreement intended to allow users to freely share, modify, and use this Dataset while maintaining this same freedom for others, provided that the original source and author(s) are credited. 


\section{Supplementary material 3}

\section{ITS/SRP RNA multiple sequence alignment}

Authors: Magnus Alm Rosenblad, María P. Martín, Leho Tedersoo, Martin Ryberg, Ellen Larsson, Christian Wurzbacher, Kessy Abarenkov, R. Henrik Nilsson

Data type: text/DNA sequence data

Explanation note: Multiple sequence alignment comprising the 63 public ITS1 sequences with SRP RNA found in them, the three newly generated sequences, and the SRP RNA sequences from Dumesic et al. (2015) (Stereum hirsutum, Heterobasidion irregulare, and Heterobasidion annosum).

Copyright notice: This dataset is made available under the Open Database License (http://opendatacommons.org/licenses/odbl/1.0/). The Open Database License $(\mathrm{ODbL})$ is a license agreement intended to allow users to freely share, modify, and use this Dataset while maintaining this same freedom for others, provided that the original source and author(s) are credited.

\section{Supplementary material 4}

\section{SRP RNA multiple sequence alignment}

Authors: Magnus Alm Rosenblad, María P. Martín, Leho Tedersoo, Martin Ryberg, Ellen Larsson, Christian Wurzbacher, Kessy Abarenkov, R. Henrik Nilsson

Data type: text/DNA sequence data

Explanation note: Multiple sequence alignment with the SRP RNA sequences of Dumesic et al. (2015; Stereum hirsutum, Heterobasidion irregulare, and Heterobasidion annosum) aligned to our newly generated ITS sequences of Russula and Lactarius.

Copyright notice: This dataset is made available under the Open Database License (http://opendatacommons.org/licenses/odbl/1.0/). The Open Database License $(\mathrm{ODbL})$ is a license agreement intended to allow users to freely share, modify, and use this Dataset while maintaining this same freedom for others, provided that the original source and author(s) are credited. 